\title{
CLINICAL EFFICACY OF DIODE LASER ALONE AND IN COMBINATION WITH DESENSITIZING VARNISH IN DENTINAL HYPERSENSITIVITY TREATMENT (RANDOMIZED CONTROLLED TRIAL)
}

\author{
Nayer aboelsaad * and Mohmed Attaf **
}

\begin{abstract}
This study aimed to evaluate the efficacy of diode laser alone and with topical sodium fluoride varnish on reducing dentine hypersensitivity $(\mathrm{DH})$ in patients with chronic periodontitis. Materials and Methods: Forty patients having stage I and Stage II periodontitis were selected according to the criteria of AAP (2017) with complaint of (DH) after routine periodontal therapy . Patients were randomly divided into two groups: group1- received $\mathrm{NaF}$ varnish then diode laser application at 1 $\mathrm{W}(\mathrm{PW}),(\mathrm{CW})$ for 30 seconds using 320 $\mu$ fiber. Group-2, treated with laser irradiation only. Each tooth received three application Dentine hypersensitivity evaluation was by tactile, air-blast, and thermal stimuli and measured using VAS scores. The patient's response was recorded at baseline, one month and 3 month after the application
\end{abstract}

Results : The results were statistically analyzed, significant pain reduction was showed in both groups. However, it was found that in the laser plus varnish group, tangible reduction in dentine hypersensitivity scores more than in laser alone group on the three tested stimuli at one and three months. VAS score differences between the groups were statistically significant

Conclusion : It was concluded that diode laser plus $\mathrm{NaF}$ varnish is more effective than laser alone in reduction of patients' dentine hypersensitivity among chronic periodontitis patients.

KEY WORDS: Diode laser, Chronic periodontitis, desensitizing agent, dentine hypersensitivity

\section{INTRODUCTION}

Dentine hypersensitivity (DH) is a quite common dental complaint that negatively impact the quality of life of some patients. ${ }^{(1)}$ Patients who may be at risk for $(\mathrm{DH})$ include: periodontal treated patients, obsessive brushers, bulimics, patients with xerostomia, high-acid food/drink regulars, elderly with gingival recession. Moreover, some dental professionals may cause dentine hypersensitivity by over instrumentation. ${ }^{(2,3)}$

\footnotetext{
*Associate Professor of Periodontology, Faculty of Dentistry, Beirut Arab University, Lebanon, Faculty of Dentistry, Mansoura University, Egypt.

** Professor of Restorative Dentistry, Faculty of Dentistry, Beirut Arab University, Lebanon .
} 
For DH development, dentin canaliculi must become exposed to the oral cavity because of gingival recessions and/or loss of enamel/cementum caused by erosion, abrasion, attrition and abfraction ${ }^{(4)}$

Pashley (5) reported that two types of dentinal permeability: intratubular and intertubular meaning into and between the dentinal tubules in dentinal matrix. Therefore ,any dental treatment to reduce dentinal permeability will lessen dentinal sensitivity. The highest dentine diffusion capacity permits the greatest interaction with desensitizing agent. Though, the DH may continue even the tubules are sealed efficiently, so indicating other process are involved in nerves activation. Many researchers hypothesised the induction of a neurogenic inflammation through release of neuropeptides from the nervous terminations . ${ }^{(6,7)}$

The dental lasers gave additional opportunities to DH management . Laser photobiomodulating effect on dental pulp was reported in the literature as Villa et al.(8) studied histologically the effect of laser irradiation on exposed dentine. The authors recorded in lased teeth physiological obliteration of tubules caused by tertiary dentine production. while inflammatory process that evolved into necrosis in few control cases.

The action of laser in DH treatment is dual. By one side, lasers act directly on nerve transmission via stoppage of pain diffusion to SNC. By the other side, aggravating a melting result with crystallization of dentine inorganic component with fluids coagulation limited to dentinal tubules. Among laser types, diode lasers gave the best treatment outcomes in even severe DH cases..$^{(9-11)}$

Some studies reported a mutual effect of lasers with desensitizing agents application. They postulated that laser favor the durability of the desensitizer for extended time instead of using alone. That's why, they recommend desensitizing agent to remain above tooth surface for one minute before laser irradiation. ${ }^{(12-14)}$
The aim of this study was to evaluate the clinical usefulness of diode laser irradiation alone and combined with topical sodium fluoride varnish $(\mathrm{NaF})$ in management of Dentinal Hypersensitivity (DH) among chronic periodontitis patients. Also, to assess duration of sensitivity relief up to three months

\section{MATERIAL \& METHODS}

This study was a, double-blind, randomized trial. The study was conducted after the approval of the Institutional Review Board (IRB) at BAU with code number 2015H-016-D-R-0050. Before intervention, patients were briefed about the study and informed consent was obtained from all participants after a detailed explanation of the safety and potential efficacy of desensitizing agents, and the probability of receiving both soft laser applications and /or $1.23 \% \mathrm{NaF}-$ varnish.

Forty patients having stage I and Stage II periodontitis where selected according to the criteria of AAP (2017) ${ }^{(15)}$ from the Department of Periodontology, faculty of dentistry -BAU. Their age (range 30-55 years) with a history of DH at least two tooth were selected.

Inclusion criteria were no less than two vital teeth with hypersensitivity on facial surfaces to three stimuli; thermal, mechanical and tactile. Nonsmokers and healthy patients were included. They complaint DH after standard periodontal therapy and did not use any hypersensitivity agents.

Exclusion criteria for teeth of the test area: caries, crowned and prosthetic restoration less than three months. Patients were randomly divided into two groups: group1- received $\mathrm{NaF}$ varnish then diode laser (Sirona) application at $1 \mathrm{~W}(\mathrm{PW})$ continues wave mode $(\mathrm{cW})$ for 30 seconds using $320 \mu$ fiber. Group-2, treated with laser irradiation only . Each tested tooth received three laser applications. (Fig.2)

Patients were instructed to use soft bristled toothbrush twice daily for two minutes before baseline examination and during the study. 


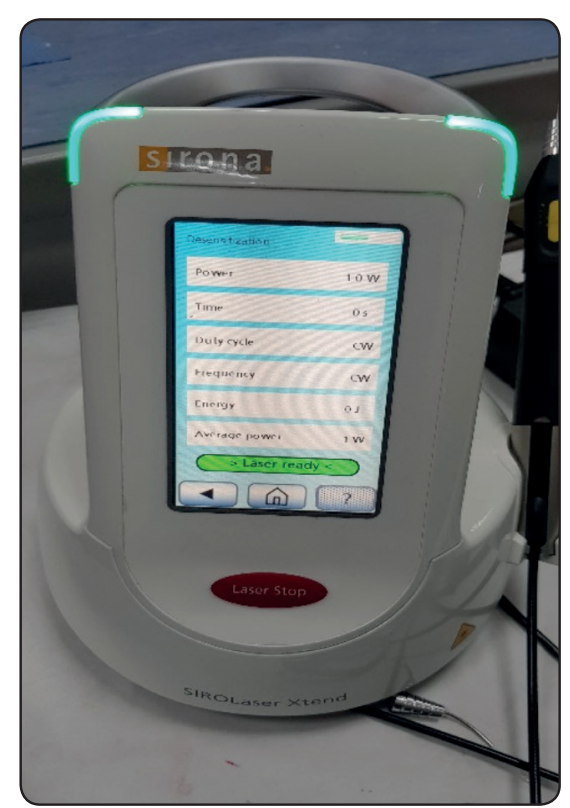

Fig. (1) Sirona Laser machine used in this study

\section{Tactile hypersensitivity}

It was done by one examiner who scratch on the dentinal surface using a sharp-tipped probe applied perpendicular to the tested tooth surfaces. Starting apical to CEJ and drawn gradually across the surface in a distal to mesial direction to confirm stimulus application across most of patent tubules.

\section{Cold water (thermal stimulus)}

After adjacent teeth isolation during testing with cotton rolls, $10 \mu \mathrm{l}$ of ice-cold water was applied to the exposed dentin surface . Five minutes was allowed between the two stimuli on each tooth.

\section{Air blast hypersensitivity}

The test surfaces were first isolated from adjacent by red boxing wax placement. Using a dental unit air syringe at maximal pressure (45 psi) and at room temperature of 20-24C. The air current was applied perpendicular to the buccal surface of the tooth for one second and at a distance of $1 \mathrm{~cm}$. The patients scored pain intensity by placing a mark on a $10 \mathrm{~cm}-$ long line on a Visual Analogue Scale (VAS) that was labeled from $(0=$ no pain to $10=$

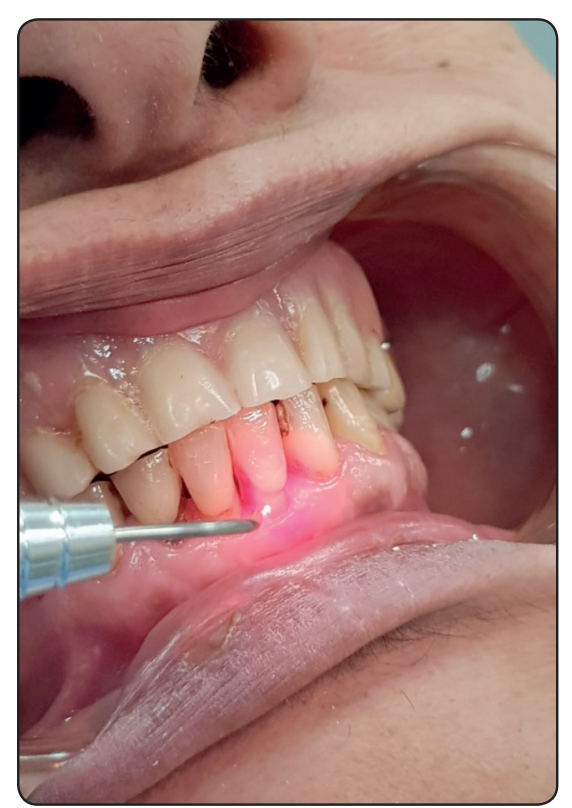

Fig. (2) Laser application on exposed root according to manufacture instructions

extreme, unbearable pain). Patients were trained to point to the VAS. ${ }^{[9,10]}$. Patients were well-informed about the different score levels before testing. Each group was evaluated at baseline; 1, and 3 months post application

\section{Statistical methods}

Within treatment using paired t-tests, comparisons of baseline versus the follow-up measurements were performed. Comparisons between treatment groups at follow up time intervals were performed using analyses of covariance (ANCOVA). Where baseline scores were employed as a co-variable. All statistical tests were two-sided, with a level of significance of 0.05 .

\section{RESULTS}

During the study, there were no adverse effects observed by the examiner or reported by the patients when questioned on both soft or hard tissues. The lateral and central incisors were the most affected tooth in both groups, followed by canines and premolars, while molars were the least affected. 
In most of the patients plaque build-up was minimal with excellent gingival condition. At first appointment, no statistically significant differences on clinical parameters between groups $(p>0.05)$

The VAS scores for the three stimulus in two groups were not statistically different at baseline $(p>0.05)$.

The laser plus $\mathrm{NaF}$ varnish group recorded better reducing VAS score for air-blast, tactile and thermal stimuli compared to the laser alone group. The changes of air-blast stimuli and thermal stimuli were significant in the laser plus $\mathrm{NaF}$ varnish group at 1 month than laser alone group $(p<0.001)$. The laser plus $\mathrm{NaF}$-gel group was more effective for tactile stimuli than laser alone group at 1 month , however the differences between groups was not statistically significant $(\mathrm{p}>0.05)$. (Table 1 )

The laser plus NaF varnish group: The differences of VAS score for the three tested stimuli were significant at baseline to 1 month $(\mathrm{p}<0.05)$. There was more reduction in sensitivity score for thermal stimuli at 1 month, followed by air-blast and tactile stimuli respectively. The mean VAS score was statistically significant at each time interval during this clinical trial for the three stimuli.

The laser alone group :The changes in VAS score of the three tested stimuli at one month were decreased in comparison with baseline, but decreasing was not statistically significant $(\mathrm{p}>0.05)$. At 3 month, the VAS scores for the three tested stimuli were lower than baseline. The differences were statistically significant.

\section{DISCUSSION}

Dentine hypersensitivity usually has multifactorial etiology and many factors commonly found active in this painful complaint ; consequently, more than one treatment modality should be applied to desensitize the dentine to acceptable levels.

Treatment of DH comprehend use of desensitizing agents applied topically by dentist or patient at home. The characters of ideal desensitizing technique/ material as easy application , non-irritating to pulp , painless, consistent and long term effectiveness and rapid acting with no discoloration. ${ }^{(16,17)}$ Moreover, recently laser irradiation become progressively popular in modern dentistry

Several authors studied the effectiveness of the diode laser alone. The success of improvement in laser-irradiated cases ranged from $60 \%$ to $95 \%$ while in nonlased group ranged about $20 \%$. $^{(18-20)}$

In double blind study reported by Gerschman et al. (21) "sensitivity to thermal and tactile stimuli was decreased by $67 \%$ in laser group. Whereas a $17 \%$ reduction in the placebo group".

Table (1) Sensitivity Scores of the three tested stimuli for both group at different time intervals.

\begin{tabular}{|c|c|c|c|c|c|c|c|c|c|}
\hline \multirow{2}{*}{ Stimuli } & \multicolumn{3}{|c|}{ Thermal } & \multicolumn{3}{c|}{ Tactile } & \multicolumn{3}{c|}{ Air Blast } \\
\cline { 2 - 10 } & Baseline & 1 month & 3 months & Baseline & 1 month & 3 months & Baseline & 1 month & 3 months \\
\hline $\begin{array}{c}\text { Laser plus } \\
\mathrm{NaF}\end{array}$ & $6.56 \pm 0.64 \mathrm{a}, \mathrm{b}$ & $4.24 \pm 0.5$ & $3.24 \pm 0.6^{*}$ & $5.79 \pm 0.6$ & $4.72 \pm 0.8$ & $3.94 \pm 0.6$ & $5.2 \pm 0.75$ & $3.4 \pm 0.7$ & $3.54 \pm 0.71^{*}$ \\
\hline Laser alone & $6.26 \pm 0.7$ & $5.5 \pm 0.4$ & $4.3 \pm 0.9^{*}$ & $5.75 \pm 0.7$ & $5.74 \pm 0.6$ & $4.44 \pm 0.7$ & $5.10 \pm 0.3$ & $4.4 \pm 0.5$ & $4.25 \pm 0.8^{*}$ \\
\hline
\end{tabular}

a "Data are expressed as mean and standard deviation".

b Difference within group according to stimulus was statistically significant $(p<0.05)$. 
It is likely that the better outcome of mutual treatment was as a result of higher $\mathrm{NaF}$ varnish bond to dentinal tubules after laser irradiation. It can be hypothesize that the laser-induced superficial melting permits to last longer occlusion of dentinal tubules emphasizing DH reducing pain.

Although ,diode lasers regularly used to treat DH. Researchers mainly emphasis on obliteration of the dentinal tubules but neglect laser's additional bio stimulatory effect. Diode laser bio-stimulation increase mitochondrial ATP. Also, it provide analgesic effect by increase in endorphins and through increases pain threshold of free nerve ending,. Moreover ,it inhibits cyclooxygenase enzyme which causes conversion of arachidonic acid into prostaglandin which in turn increases the pain transmission. In addition, laser biostimulation stimulate formation of secondary dentin by odontoblast and simultaneously reduce inflammation ${ }^{(15-19)}$.

In agreement to our results, Wakabayashi and Matsumoto (8) "reported that diode laser was effective in 61 of 66 cases. The combined use of the $830 \mathrm{~nm}$ wavelength diode laser with fluoridation boosts treatment effectiveness by more than twenty percent over that of laser treatment only".

Moreover, some researcher reported that low power laser irradiation promoted significantly better treatment outcome, launching an irradiation protocol of three applications with more favorable results $\cdot(10,20)$

Within the limitation of this study, results need to be long-established by longer periods (e.g one year) and greater number of patients to in order to check the continuing effect of the mutual laser and desensitizing agent application.

\section{CONCLUSION}

Based on this research results, the diode laser both alone and moreover better in combination with $\mathrm{NaF}$ varnish proved capability to improve instantly the dentinal hypersensitivity associated pain. Combined with conventional desensitizing agents, diode laser irradiation considered valuable treatment modality in modern dentistry.

\section{REFERENCES}

1. Praveen R, Thakur S, Kirthiga M, Narmatha $M$. Comparative evaluation of a low-level laser and topical desensitizing agent for treating dentinal hypersensitivity: A randomized controlled trial. J Conserv Dent. 2018 SepOct;21(5):495-499

2. Lopes AO, Eduardo Cde P, Aranha AC. Clinical evaluation of low-power laser and a desensitizing agent on dentin hypersensitivity. Lasers Med Sci. 2015 Feb;30(2):823-9.

3. Gojkov-Vukelic M, Hadzic S, Zukanovic A, Pasic E, Pavlic V . Application of Diode Laser in the Treatment of Dentine Hypersensitivity. Med Arch. 2016 Dec;70(6):466469.

4. El Mobadder M, Namour A, Namour M, Dib W, El Mobadder W, etal,. Dentinal Hypersensitivity Treatment Using Diode Laser 980 nm: In Vivo Study. Dent J (Basel). 2019 Jan 9;7(1). pii: E5.

5. Pashley D.H., "Dentin permeability and dentin sensitivity," Proceedings of the Finnish Dental Society, vol. 88, pp. 3137,1992

7. S. Pesevska, M. Nakova, K. Ivanovski et al., "Dentinal hypersensitivity following scaling and root planing: comparison of low-level laser and topical fluoride treatment," Lasers in Medical Science, vol. 25, no. 5, pp. 647-650, 2010.

8. R. Orchardsonand D.G. Gillam, "Managing dentin hypersensitivity," Journal of the American Dental Association, vol. 137, no. 7, pp. 990-998, 2006.

9. S. D. Ipci, G. Cakar, B. Kuru, and S. Yilmaz, "Clinical evaluation of lasers and sodium fluoride gel in the treatment of dentine hypersensitivity," Photomedicine and Laser Surgery, vol. 27, no. 1, pp. 85-91, 2009

8. H. Wakabayashi and K. Matsumoto, "Study on the treatment of hypersensitive dentine by laser," Journal of Conservative Dentistry, vol. 28, pp. 1366-1371, 1985

9. Yamaguchi, M. Ito, T. Miwata et al., "Clinical study on the treatment of hypersensitive dentin by GaAlAs laser 
diode using the double blind test," Aichi Gakuin Daigaku Shigakkai shi,vol. 28, no. 2, pp. 703-707, 1990

10. Machado AC, Viana ÍEL, Farias-Neto AM, Braga MM, Is photobiomodulation (PBM) effective for the treatment of dentin hypersensitivity? A systematic review. Lasers Med Sci. 2018 May;33(4):745-753.

11. W. H. Lan, H. C. Liu, and C. P. Lin, "The combined occluding effect of sodium fluoride varnish and Nd:YAG laser irradiation on human dentinal tubules," Journal of Endodontics, vol. 25, no. 6, pp. 424-426, 1999.

12.Pourshahidi S, Ebrahimi H, Mansourian A, Mousavi Y, Kharazifard M. Comparison of Er,Cr:YSGG and diode laser effects on dentin hypersensitivity: a split-mouth randomized clinical trial. Clin Oral Investig. 2019 Feb 18. doi: 10.1007/s00784-019-02841-z. [Epub ahead of print]

14. A. Sicilia, S. Cuesta-Frechoso, A. Su'arez, J. Angulo, A.et al, "Immediate effcacy of diode laser application in the treatment of dentine hypersensitivity in periodontal maintenance patients: a randomized clinical trial," Journal of Clinical Periodontology, vol. 36, no. 8, pp. 650-660, 2009.

15. Papapanou PN, Sanz M, Buduneli N, Dietrich T, Feres M, Fine DH, et al,. Periodontitis: Consensus report of workgroup 2 of the 2017 World Workshop on the Classification of Periodontal and Peri-Implant Diseases and Conditions. J Periodontol. 2018 Jun;89 Suppl 1:S173-S182.
16. S. A. M. Corona, T. N. Do Nascimento, A. B. E. Catirse, R. F. Z.,et al "Clinical evaluation of low-level laser therapy and fluoride varnish for treating cervical dentinal hypersensitivity," Journal of Oral Rehabilitation, vol. 30, no. 12, pp. 1183-1189, 2003

17. Osmari D, Fraga S, Ferreira ACO, Eduardo CP, Marquezan M, Silveira BLD. In-office Treatments for Dentin Hypersensitivity: A Randomized Split-mouth Clinical Trial. Oral Health Prev Dent. 2018;16(2):125-130.

18. Soares ML, Porciúncula GB, Lucena MI, Gueiros LA, Leão JC, Carvalho AA. Efficacy of Nd:YAG and GaAlAs lasers in comparison to $2 \%$ fluoride gel for the treatment of dentinal hypersensitivity. Gen Dent. 2016 NovDec;64(6):66-70.

19. Sgolastra F, Petrucci A, Severino M, Gatto R, Monaco A. Lasers for the treatment of dentin hypersensitivity: a metaanalysis. J Dent Res. 2013 Jun;92(6):492-9.

20. Lopes AO, de Paula Eduardo C, Aranha ACC. Evaluation of different treatment protocols for dentin hypersensitivity: an 18-month randomized clinical trial. Lasers Med Sci. 2017 Jul;32(5):1023-1030.

21. J. A. Gerschman, J. Ruben, and J. Gebart-Eaglemont, "Low level laser therapy for dentinal tooth hypersensitivity," Australian Dental Journal, vol. 39, no. 6, pp. 353-357, 1994. 